\title{
INVESTIGATION OF GRAPE SEED PROANTHOCYANIDINS. ACHIEVEMENTS AND PERSPECTIVES ${ }^{\S}$
}

\author{
V. Kulcițki, P. F. Vlad*, Gh. Duca, T. Lupaşcu \\ Institutul de Chimie al Academiei de Ştiințe a Moldovei, str. Academiei 3, MD-2028, Chişinău, Republic of Moldova \\ *E-mail: vlad_p@mail.md; Phone: 37322 739775; Fax: 37322739775
}

\begin{abstract}
The present paper provides an account of the basic technuques employed in the investigation of the grape seeds proanthocyanidins (condensed tannins). The importance and biological activity properties of these compounds are considered briefly in the introductory part, while isolation and structural investigation of grape seeds proanthocyanidins represent the basic part of the review. The references cover mostly the recent publications related to implementation of modern techniques of investigation, like high performance liquid chromatography (HPLC) and mass spectrometry (MS). Number of references -68 .
\end{abstract}

Keywords: polyphenols, proanthocyanidins, grape seeds, chromatography, MS, NMR.

\section{Content}

1. Introduction. Grape seeds tannins, biological activity and applications

2. Chemical structure of proanthocyanidins

3. Extraction methods employed in the grape seed tannins isolation

4. Methods for proanthocyanidins fractionation

5. Chemical methods in the investigation of proanthocyanidins

6. Phisico-chemical methods in the investigation of proanthocyanidins HPLC methods Mass-spectrometry methods

Miscellaneous methods

7. Conclusions

8. References

\section{INTRODUCTION. GRAPE SEEDS TANNINS, BIOLOGICAL ACTIVITY AND APPLICATIONS.}

Grape seeds condensed tannins (GST), called also proanthocyanidins, represent a group of natural products with polyphenolic structure. The interest towards these compunds is determined by several factors. First of all they posess a wide spectrum of biological activity, and their presence in the human diet is considered a preventing measure to avoid such severe pathologies like stroke and cancer. The beneficent intake of grape seeds tannins by humans is connected with moderate wine consumption. Grape seeds represent a byproduct of grape processing and they contain appreciable amounts of polyphenolic compounds. Provided the fact that valorification of the wine industry secondary products is one of the research priority directions recognized by Moldovan governement, several companies developed research programs directed to elaboration of efficient procedures for proanthocyanidins extraction from the grape seeds.

Historically, the first explored source of condensed tannins was the pine bark. One of the most preferable sources of these compounds in the recent years became the grape seeds, which are readily available in industrial quantities and a lot of different commercial grape seeds extracts are on the market as sources of grape seeds proanthocyanidins. Tannins are highly hydroxylated aromatic structures that form insoluble complexes with carbohydrates and protein, a measure of their astringency, based on their ability to cause precipitation of salivary proteins. The polyphenolics, including proanthocyanidins, constitutes a considerable portion of the tannins found in wine, and in particular contribute heavily to the colour and flavour of red wines.

Oligomeric proanthocyanidin complexes are primarily known to be responsible for organolepthic properties of wine, including colour and astringency, ${ }^{1,2,3,4}$ Besides, derivatives of flavan-3-ol are important natural antioxidants in living cells. Therefore, grape seeds proanthocyanidins are considered for their antioxidant activity first. The redoxpotential of the phenolics is known to be sufficiently low, to interact easily with different oxidyzing species by different mechanisms, basically by a radical scavenging one. ${ }^{5,6}$ 
These compounds can also play an important role as repellents in plant protection against pathogens and different predators. ${ }^{3}$ Literature data show that proanthocyanidins effect a protective action on DNA oxidative damage and have a benefical action to the preventive carcinogenesis inhibition. ${ }^{7}$ Along with their antioxidant properties, proanthocyanidins can influence the release of nitrogen oxide by endothelial cells. ${ }^{8}$ Basing on these mechanisms of action, these compounds have been reported to demonstrate antibacterial, antiviral, anticarcinogenic, anti-inflammatory, anti-allergic and vasodilatory actions. In addition, they have been reported to inhibit platelet aggregation, improve capillary permeability, diminish their fragility and to affect enzyme systems including phospholipase A2, cyclooxygenase, and lipoxygenase. The potential health benefit of proanthocyanidins was broadly recognized. ${ }^{9}$ These varied biological activities have resulted in the phytopharmaceutical application of condensed tannins in reduction of edema, increased peripheral circulation, improvement in vision, treatment of diabetic retinopathy, prevention of cardiovascular disease, treatment of hypercholesterolemia, stabilization of connective tissue tone, reduced adverse allergic and inflammatory responses, and enhanced immune function and wound healing. ${ }^{10}$ The well mediated "French paradox" ${ }^{11}$ consists in the lower incidence of cardiovascular diseases, including fatal cases in France, compared with similar data for Great Britain or USA. It is explained by the regular wine consumption in France, that contributes to chronic inflammation inhibition and antiplatelet activity. ${ }^{12}$

Grape seeds proanthocyanidin extracts are used as active pharmaceutical components in different preparations (e.g. Leucoselect TM) for treatment of blood vessels fragility, increasing their walls stability, as well as for free radical quenching and superoxide ions inhibition. ${ }^{13}$

Basing on these data, grape seeds proanthocyanidins are of great interest as biologically active compounds with a broad spectrum of activity.

\section{CHEMICAL STRUCTURE OF GRAPE SEED TANNINS}

The composition of the grape seeds proantocianidins is very complex. The structural units that are on the basis of this complexity are two polyfenolic compounds: catechin 1 and epi-catechin $\mathbf{2}$.<smiles>Oc1ccc([C@@H](Oc2ccc(O)c(O)c2)C(O)Cc2c(O)cc(O)cc2O)cc1</smiles><smiles>Oc1cc(O)c2c(c1)O[C@H](c1ccc(O)c(O)c1)[C@H](O)C2</smiles>

Oxidative condensation occurs between carbon C-4 of 1 or 2 and carbons C-6 or C-8 of the attached molecules. The resulting oligomers form a plethora of compounds differing in degree of polymerization (DP), linkage patterns and monomers sequence. Procyanidins B1-B4 (3-6), having the C4-C8 linkage, are the most common dimers. ${ }^{14}$ Some oligomers and polymers have the C-3 hydroxil group esterified with galic acid. ${ }^{8,14}$<smiles>[R2]C1([R1])Cc2c(O)cc(O)c([C@@H]3c4c(O)cc(O)cc4O[C@H](c4ccc(O)c(O)c4)[C@H]3O)c2O[C@H]1c1ccc(O)c(O)c1</smiles><smiles>[R1]C1([R1])Cc2c(O)cc(O)c([C@@H]3c4c(O)cc(O)cc4O[C@H](c4ccc(O)c(O)c4)[C@H]3O)c2O[C@H]1c1ccc(O)c(O)c1</smiles>

$3 \mathrm{R}_{1}=\mathrm{OH} ; \mathrm{R}_{2}=\mathrm{H}$ Proanthocyanidin $\mathrm{B} 1 \quad 5 \mathrm{R}_{1}=\mathrm{OH} ; \mathrm{R}_{2}=\mathrm{H}$ Proanthocyanidin $\mathrm{B} 3$ $4 \mathrm{R}_{1}=\mathrm{H} ; \mathrm{R}_{2}=\mathrm{OH}$ Proanthocyanidin $\mathrm{B} 2 \quad 6 \mathrm{R}_{1}=\mathrm{H} ; \mathrm{R}_{2}=\mathrm{OH}$ Proanthocyanidin B4

It is noteworthy mentioning that only few individual pure proanthocyanidins were isolated and characterized, ranging in structure up to tetramers. ${ }^{13}$ Distribution of proanthocyanidins in grape seeds, including of white grapes, was studied by many authors. ${ }^{13,15,16-19}$ Monomeric flavanols and their galates predominates in the water soluble procyanidins fraction obtained from grape seeds. Oligomers are represented mostly by B-type proanthocyanidins. ${ }^{18,20}$ Their structure was investigated in the works. ${ }^{11,21,22}$ A comparative study of flavan-3-ols composition was performed. ${ }^{20}$ They were isolated from the seeds of 17 different varieties of Spanish grapes, including 10 red and 7 white samples. A total number of 27 proanthocyanidins were identified: (+)-catechin, (-)-epi-catechin, epi-catechin-3-O-galate, dimmers B-1 - B-7 (3)- 
(9), trimers C-1 (11) and C-2 (12), along with a bunch of proanthocyanidins esterified with galic acid on C-3 hydroxyl group in B-cycle and on C-3' phenolic hydroxyl in C-cycle.<smiles>[R2]C1([R2])Cc2c(cc(O)c([C@@H]3c4c(O)cc(O)cc4O[C@H](c4ccc(O)c(O)c4)[C@H]3O)c2O)O[C@H]1c1ccc(O)c(O)c1</smiles>

$7 \mathrm{R}_{1}=\mathrm{H} ; \mathrm{R}_{2}=\mathrm{OH}$ Proanthocyanidin $\mathrm{B} 5$

$8 \mathrm{R}_{1}=\mathrm{OH} ; \mathrm{R}_{2}=\mathrm{H}$ Proanthocyanidin $\mathrm{B} 7$<smiles>Oc1cc(O)c2c(c1)O[C@H](c1ccc(O)c(O)c1)[C@]2(O)c1c(O)cc(O)c2c1O[C@H](c1ccc(O)c(O)c1)[C@H](O)[C@H]2c1c(O)cc(O)c2c1O[C@H](c1ccc(O)c(O)c1)[C@H](O)C2</smiles>

11 Proanthocyanidin C1<smiles>[R2]C1([R2])Cc2c(cc(O)c([C@H]3c4c(O)cc(O)cc4O[C@H]3O)c2O)O[C@H](c2ccc(O)c(O)c2)[C@@H]1c1ccc(O)c(O)c1</smiles>

$9 \mathrm{R}_{1}=\mathrm{OH} ; \mathrm{R}_{2}=\mathrm{H}$ Proanthocyanidin $\mathrm{B} 6$
$10 \mathrm{R}_{1}=\mathrm{H} ; \mathrm{R}_{2}=\mathrm{OH}$ Proanthocyanidin B8<smiles>Oc1cc(O)c2c(c1)O[C@H](c1ccc(O)c(O)c1)[C@H](O)[C@H]2c1c(O)cc(O)c2c1O[C@H](c1ccc(O)c(O)c1)[C@H](O)[C@H]2c1c(O)cc(O)c2c1O[C@H](c1ccc(O)c(O)c1)[C@H](O)C2</smiles>

12 Proanthocyanidin C2

Among esterified compounds there are dimers and trimers of flavan-3-ol, some of them contain two galic acid residues. The B-2 dimer (4) predominates in the dimeric group. The described proanthocyanidins differ not only by the number and sequence of flavan-3-ol units, esterification patern, but also by different monomer junction (C-4 C-8 and C-4 - C-6) and C-4 configuration ( $\alpha$ or $\beta$ ). There is only one described tetramer in this work and it consists

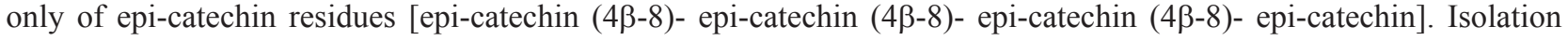
of individual polymeric tannins represents a very difficult task. All of the reported examples relate only isolation of fractions containing mixtures of several polymers. ${ }^{13}$

The mean degree of polymerisation (mDP) of flavan-3-ols can be different and the obtained data depend on the determination method. According to authors ${ }^{1}$ high molecular weight proanthocyanidins can contain up to 20 flavan3 -ol units and these compounds are soluble in wine. A trigaloilated octamer was also reported. ${ }^{23}$ Mass-spectrometric investigations ${ }^{24}$ reported the polymerisation degree from 9 to 11 units. In the same time, basing on acidic hydrolysis experiments ${ }^{25,26}$ higher values of 17 and 31 units were reported. A more detailed analysis of these data will be provided in the following discussion below.

\section{EXTRACTION METHODS EMPLOYED IN THE GRAPE SEEDS TANNINS ISOLATION}

Isolation of grape seeds proanthocyanidins includes seed grinding followed by extraction with a suitable solvent. Different solvents have been employed: acetone, ${ }^{27,28}$ acetone-water mixture 70:30 in the presence of silicagel, ${ }^{15} 60: 40$ at $+4{ }^{\circ} \mathrm{C},{ }^{1} 50: 50$ at room temperature, ${ }^{2}$ methanol containing ascorbic acid $(1 \mathrm{~g} / \mathrm{l})$ at $-20^{\circ} \mathrm{C}$, followed by methanol-water 
mixture $(1: 1)$ at $-24^{\circ} \mathrm{C}$ and acetone-water mixture $(75: 25)$ all under nitrogen atmosphere. ${ }^{20,29}$ Methanol in combination with other solvents was used for extraction of tannins too. Some examples include extraction with HCl-acidified methanol, ${ }^{30}$ methanol-water mixture (75:25) at room temperature under ultrasound irradiation, ${ }^{3}$ pure methanol at room temperature. ${ }^{8}$ Aqueous ethanol was used for extraction of grape seeds tannins ${ }^{5,31}$ and proanthocyanidins from Rhodiola semenovii. ${ }^{32}$

Proanthocyanidins are unstable compounds. They undergo facile oxidation by atmospheric oxygen. That's why some authors used antioxidants $\left(\mathrm{SO}_{2}\right.$, ascorbic acid) dissolved in extraction solvents employed in the grape seeds extraction. Other preventive actions include extraction under inert atmosphere $\left(\mathrm{N}_{2}\right)$, as well as grinding of the raw material under liquid nitrogen. ${ }^{1}$ According to authors, ${ }^{11}$ the mixture acetone-water is the best extraction solvent for grape seeds proanthocyanidins. Nevertheless, all of the enumerated solvents have the drawback to extract along with proanthocyanidins other accompanying compounds that make extraction laborious and lower the proanthocyanidins yield. There are no literature data reporting on selective solvent systems for proanthocyanidin extraction. On the other hand, it is known that lower molecular weight catechin oligomers are soluble in ethylacetate and this solvent showed notable selectivity for the extraction of some other natural compounds. In this context, authors ${ }^{11}$ studied the extraction of grape seeds with ethylacetate as a method of preparative isolation of proanthocyanidins. It is noteworthy mentioning that the grape seeds were extracted without grinding, as a necessary condition to avoid concomitant extraction of ballast compounds. It turned out, though, that ethylacetate practically does not extract proanthocyanidins, due to a very low permeability of seeds wall for the non polar aprotic solvent. The solution of the problem included extraction with the mixture ethylacetate-water (90:10) and this mixture provided a selective extraction of proanthocyanidins according to a simple procedure which allowed preparative extractions in industrial quantities.

Another interesting extraction example ${ }^{32}$ includes fractionation of an ethanol-water extract by successive extraction with diethyl ether and ethylacetate to provide more simple proanthocyanidin fractions. The etheric fraction included (+)-catechin (1), (+)-gallocatechin (13), (-)-epi-catechin (2), (-)-epi-catechin-gallate (14), (-)-epi-gallocatechin (15), (-)-epi-gallocatechin-gallate (16) and gallic acid (17). The ethylacetate fraction contained the following dimers:

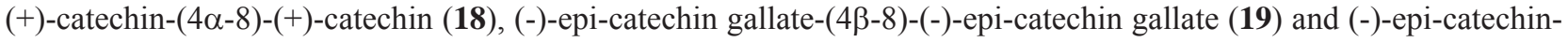

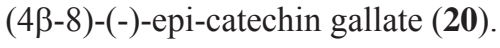<smiles>[R2]c1cc([C@H]2Oc3cc(O)cc(O)c3CC2[R1])cc(O)c1O</smiles>

$13 \mathrm{R}_{1}=\beta-\mathrm{OH} ; \mathrm{R}_{2}=\mathrm{OH}$

$14 \mathrm{R}_{1}=\alpha-\mathrm{OGalloyl} ; \mathrm{R}_{2}=\mathrm{H}$

$15 \mathrm{R}_{1}=\alpha-\mathrm{OH} ; \mathrm{R}_{2}=\mathrm{OH}$

$16 \mathrm{R}_{1}=\alpha-\mathrm{OGalloyl} ; \mathrm{R}_{2}=\mathrm{OH}$<smiles>Oc1cc(O)c2c(c1)O[C@H](c1ccc(O)c(O)c1)[C@H](O)[C@H]2c1c(O)cc(O)c2c1O[C@H](c1ccc(O)c(O)c1)[C@H](O)C2</smiles>

18<smiles>O=C(O)c1cc(O)c(O)c([12F])c1</smiles><smiles>[R1]C1[C@@H](c2ccc(O)c(O)c2)Oc2cc(O)cc(O)c2[C@H]1c1c(O)cc(O)c2c1O[C@@H](c1ccc(O)c(O)c1)C([R2])C2</smiles>

$19 \mathrm{R}_{1}=\mathrm{R}_{2}=\alpha$-OGalloyl

$20 \mathrm{R}_{1}=\alpha-\mathrm{OH} ; \mathrm{R}_{2}=\alpha-O G$ alloyl

\section{METHODS FOR PROANTHOCYANIDINS FRACTIONATION}

Semipreparative fractionation of proanthocyanidins extracts is normaly performed by column chromatography. The separation procedure is based on the gel filtration-gel permeation chromatography (GPC). As the stationary phase Sephadex LH-20 is used most of all. For example Oszmianski and collaborators ${ }^{15}$ used a column with Sephadex suspended in $96 \%$ ethanol. Elution of the column was performed with the same solvent. For a successful resolution of (+)-catechin and (-)-epichatechin the column was filled with a Sephadex slurry in water and elution was performed with a solution of acetic acid with the gradual increasing the acid concentration from 0 to $25 \%$. Monitoring of the column eluate was performed by UV detection at $280 \mathrm{~nm}$. The further investigation of the obtained fractions was performed by HPLC. Degree of polymerisation of individual fractions was estimated by TLC. It has been noted that the mean degree of polymerisation can be established by gel filtration. In the other work, investigation of Leucoselect ${ }^{\mathrm{TM}} 13$ included dissolution of the sample in $90 \%$ ethanol, followed by fractionation on Sephadex LH-20. Elution was started with the same solvent, followed by a stepwise gradient of acetone in water $(20 \%, 40 \%, 70 \%$ acetone). The obtained fractions were studied further by TSI/ESI MS, HPLC (GPC). A Sephadex column was used also for the fractionation of proanthocyanidins from Rhodiola Semenovii. ${ }^{32}$ Fractionation of the mixture of proanthocyanidins on Sephadex was used 
in other works too. ${ }^{3,7}$ It is noteworthy mentioning that in the latter work ${ }^{7}$ authors used the proanthocyanidin separation methodology described in earlier works. ${ }^{33,34}$

Fractionation of proanthocyanidins extract was performed also on columns with polyamide. . $^{29,35}$ The TLC-grade polyamide was used for column chromatography in the work, ${ }^{35}$ while commercial Macherey-Nagel, Duran (Germany) polyamide was used in the work. ${ }^{29}$

Preliminary fractionation of the grape seed extracts was based on adsorption chromatography using commercial Toyopearl TSK HW-40(F) gel (Tosoh corp., Tokyo) ${ }^{8,1,2}$ The separation procedure has been described in the earlier work. ${ }^{36}$ Monomers and oligomers have been eluted from the column with a mixture ethanol-water (55:45, v/v), containing $0.05 \%$ trifluoroacetic acid and polymeric proanthocyanidins with a mixture ethanol-water $(60: 40, \mathrm{v} / \mathrm{v}) .{ }^{1}$ Fractogel TSK HW-40 (s) was used to fractionate a proanthocyanidin extract. ${ }^{5}$ The origin of the stationary phase was not specified. Microcrystallic cellulose powder was also used for the column chromatography of proanthocyanidines. ${ }^{32}$

One should mention that the methodologies related to the separation of non-polar phenols from polymeric ones have been paid a lot of attention in the literature. But most of the methods are rather complicated, time consuming and lead to substantial amount of wastes. On the contrary, a very simple method has been elaborated on the basis of solid phase extraction (SPE) that allows a quick and efficient separation of phenolic compounds from grapes. ${ }^{25}$ The method consists of the application of the sample solution on to a SPE cartridge (e.g. $\mathrm{C}_{18}$ Sep-Pak), followed by elution with solvents of different polarity. Low molecular weight components are separated from oligomers and higher polymers. A fractionation example of preliminary adsorbed on a SPE cartridge consists in elution of monomeric falavonols with diethyl ether and oligomeric with polymeric proanthocyanidins with methanol. ${ }^{1}$ Authors ${ }^{29}$ eluted from the SPE cartridge the phenoloxiacids with an aqueous buffer $(\mathrm{pH}=7)$, then monomeric and oligomeric flavan-3-ols have been eluted with ethylacetate, followed by a final wash with methanol to remove the polymeric proanthocyanidins. The fraction containing monomers and oligomeric flavan-3-ols was applied to SPE repeatedly to elute monomers with diethyl ether and oligomers with methanol. A similar fractionation of proanthocyanidines from wine was reported to produce 3 fractions of different polarity. ${ }^{7}$ Phenoloxiacids were eluted with water, catechins, flavonols and anthocyanidins with ethylacetate while polymeric compounds were eluted with a mixture of methanol/acetone/water.

Fractions obtained after the preliminary fractionation are subjected as usual to a more rigorous separation procedure by HPLC methods.

\section{CHEMICAL METHODS IN THE INVESTIGATION OF PROANTHOCYANIDINS}

Chemical methods of investigation have been among the first approaches in the investigation of the chemical structure of complex polymeric polyphenolics from grape seeds. Most of these methods comprise a degradation of the polymeric structure under acidic conditions in the presence of a nucleophile. As the result, valuable information concerning proanthocyanidine structure can be obtained, including the mean degree of polymerisation, nature of monomeric flavanols and terminal groups. As nucleophilic agents benzylmercaptane $e^{1,29,5,30,37-39}$ and floroglucinol ${ }^{40}$ have been used in most of the investigations. In the case of the benzylmercaptane treatment extender units of the oligomer I provide flavan-3-ols thyoethers II and flavan-3-ols III from the terminal units. The mechanism of this cleavage is represented in the scheme below. Initial protonation of the pyran ring is followed by the nucleophilic attack of the sulphur compound and $\mathrm{C} 4-\mathrm{C} 8$ bond cleavage.<smiles>COc1cc([C@@H]2Oc3c(c(O)cc(O)c3[C@@H]3c4c(O)cc(O)c(C)c4O[C@H](c4ccc(O)c(O)c4)[C@H]3O)C[C@H](O)[C@@H]2O)ccc1O</smiles>

I

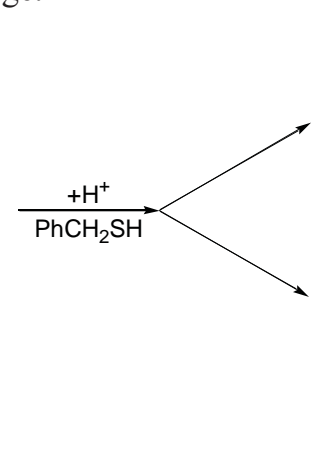<smiles>Cc1c(O)cc(O)c2c1O[C@H](c1ccc(O)c(O)c1)[C@H](O)[C@H]2[Si]=[I]</smiles><smiles>Oc1cc(O)c2c(c1)O[C@H](c1ccc(O)c(O)c1)[C@H](O)C2</smiles>

After acidic treatment in the presence of benzylmercaptane the reaction product can be treated with Raney-Ni ${ }^{5}$ in order to remove the sulphur residue. Similar data are obtained after acidic hydrolysis in the presence of floroglucinol. The reaction is performed in methanol in the presence of sulphuric acid and excess floroglucinol. The method provide information on the flavan-3-ol units composition, conversion yield and mean degree of polymerisation. ${ }^{3,40}$

Proanthocyanidin cleavage was also performed under enzymatic catalysis, using phenol heterosidase. ${ }^{5}$ The hydrolizate is analysed successively by HPLC. Proanthocyanidin structure was also evaluated by acidic 
depolymerisation using hydrochloric acid in butanol, ${ }^{41}$ total phenolics was determined by the well known FolinCiocalteau method and some other less common methods were also used. But all of the approaches lack reproducibility and specificity, due to the complex nature of the analytical matrix and side reactions that have been also reported. ${ }^{13}$

\section{PHISICO-CHEMICAL METHODS IN THE INVESTIGATION OF PROANTHOCYANIDINS}

Investigation of the grape seed condensed tannins following after rough fractionation was reported by different phisico-chemical methods of analysis. Classical chemical methods of investigation were followed by modern techniques, such as chromatographic (TLC, GLC, HPLC) and spectroscopic (UV-Vis, IR, MS, NMR) methods. But due to the high complexity of the studied mixtures of compounds, the most important information about the proanthocyanidin structure was obtained by HPLC and MS methods. Additionally, high field NMR spectroscopy has become also a powerful investigation tool, provided the fact that high performance NMR spectrometers (500 $\mathrm{MHz}$ and higher) has become routine equipment in modern laboratories. Basing on these considerations we will discuss below these modern methods of investigation and their implementation in the field of grape seeds condensed tannins chemistry.

\subsection{HPLC methods}

Along with the spectacular advent of HPLC, it was broadly use in the analysis of polyphenols of different botanical origins, including condensed tannins from wine, grapes and grape seeds. The power of this analytical method consists of the possibility to resolve highly polar compounds at ambient conditions, with no need of obtaining derivatives or sample heating. In the last decade several improvements have been made to the HPLC equipment which busted efficiently the overall method performance. First of all it is noteworthy mentioning the introduction of Diode Array Detectors in the UV-Vis monitoring of the separation process. They made it possible simultaneous monitoring of the column eluate on the whole spectral width, making possible "on the fly" registering of UV spectra of individual peaks and peak sections. This is a valuable information for peak identification and peak purity estimation,

The other breakthrough in HPLC is connected to the establishment of the interface between the HPLC and the mass spectrometer. It was possible to implement this hyphenated technique due to the development of efficient electrospray ionization methods and narrow bore columns that reduced substantially the flow rates and peak volumes. In such a way the HPLC-MS method became the most versatile analytical technique for compounds ranging from low molecular weight to biopolymers.

Basing on these achievements, HPLC has found extensive applications in different areas of natural products research, including investigation of grape polyphenols. Two early pioneers of HPLC in oenology were Nagel and Wulf, who published a series of articles that were among the first applications of HPLC to phenolics analysis. ${ }^{42-44}$ While initial HPLC studies tended to emphasize the novelty of the method, particularly its speed, oenologists soon began to apply the technique in monitoring the wine quality, including changes in flavonoid content during fermentation and aging of red wines. ${ }^{44}$ The application of HPLC to the procyanidin content of ciders and wines was also reported, ${ }^{45,46}$ as well as the anthocyanidin profile in wines across 10 years of aging. ${ }^{47}$

A comprehensive review on the HPLC of food flavonoids, including proanthocyanidins, has been published. ${ }^{48}$ This review covers the period until 1999 but after that a lot of new papers relating HPLC separation of grape proanthocyanidins has been published.

For proanthocyanidins in foods, analysis by RP chromatography often has been the primary method of choice. HPLC-UV quantitative analysis of proanthocyanidins is typically carried out at $280 \mathrm{~nm}$. However, UV detection is not specific for proanthocyanidins relative to other polyphenolic compounds. Fluorescence detection (excitation 276 and emission $316 \mathrm{~nm}$ ) offers increased sensitivity and selectivity to procyanidins. ${ }^{49} \mathrm{RP}$ C18 columns have been used to separate monomers to trimers in the Spanish diet, ${ }^{50}$ to tetramers in wine ${ }^{51}$ and grape seed. ${ }^{52,53}$ Proanthocyanidin oligomers do separate based on their degree of polymerization (monomers through tetramers) and as individual compounds, however, order of elution is not in accordance with molecular size. Furthermore, analysis beyond tetramers has not been achievable with RP chromatography because of retention time overlap and co-elution of higher oligomeric isomers.

Many reports relating to HPLC analysis of GST is connected to the investigation of their biological activity. Fitzpatrick and collaborators describe the isolation from grape seeds and characterization of some of the compounds responsible for endotheliumdependent relaxation of blood vessels activity ${ }^{8}$. Concord grape seeds were extracted with methanol and the compounds were analyzed by analytical and semipreparative HPLC after preliminary fractionation. A Waters HPLC system was employed in conjunction with a $481 \mathrm{UV} /$ Vis detector, Radial Pak reverse phase NovaPak C18 column, protected by a guard column of the same material. Elution was performed in the gradient mode, employing two 
solvents. Mobile phase A was water, mobile phase B was $10 \%$ acetic acid in water. The gradient ran from $25 \%$ B up to $100 \% \mathrm{~B}$ isocratic over $55 \mathrm{~min}$ according to a complex profile. Flow rate was $1.0 \mathrm{~mL} / \mathrm{min}$ and detection was made at 280 $\mathrm{nm}$. The integral extracts showed a complex profile, with few well resolved identified peaks, except those of monomeric gallic acid, epicatechins and epicatechin galate. One fraction obtained after gel-permeation chromatography showed a satisfactory resolution of compounds possessing increased activity, and from this fraction an individual compound was isolated in a semi-preparative experiment. According to MS data it was a galloylated trimer, and the tentative structure 21 was proposed basing only on MS-MS experiment. Further investigations are necessary, in order to ascertain the stereochemistry of this proanthocyanidin compound.<smiles>O=C(O[C@H]1Cc2c(O)cc(O)c([C@@H]3c4c(O)cc(O)c([C@@H]5c6c(O)cc(O)cc6O[C@H](c6ccc(O)c(O)c6)[C@H]5O)c4O[C@H](c4ccc(O)c(O)c4)[C@H]3O)c2O[C@H]1c1ccc(O)c(O)c1)c1cc(O)c(O)c(O)c1</smiles>

Leucoselect ${ }^{\mathrm{TM}}$ grape seed selected proanthocyanidins were analyzed by Peterlongo and collaborators ${ }^{13}$. HPLC - thermospray mass spectrometry allowed the detection of monomeric flavan-3-ols and dimeric proanthocyanidins in separate fractions of the commertial product obtained after GPC on Sephadex ${ }^{\circledR}$ LH-20. Separations were performed at room temperature on a SupelcoSil LC-18 column $(250$ x $4.6 \mathrm{~mm}$ i.d., particle size $5 \mu \mathrm{m})$. The solvent system was a linear gradient using $\mathrm{MeCN}$ (solvent A) and 0.3\% phosphoric acid (solvent B) from 10\% A to 60\% A (complex profile) in $65 \mathrm{~min}$. The flow-rate was $0.7 \mathrm{ml} / \mathrm{min}$, the UV detector was set at $278 \mathrm{~nm}$ and the injection volume was $10 \mu \mathrm{l}$. Higher oligomers gave an unresolved band under these conditions.

A satisfactory resolution of GST up to trimers was reported on the HPLC of wine-like models ${ }^{1}$. Direct HPLC analyses were performed using a Waters Millenium HPLC-DAD system The column consisted of a Merck reversedphase Lichrospher 100-RP 18 (250 $\mathrm{mm}$ x $4 \mathrm{~mm}$ i.d.) protected with a guard column of the same material and was equilibrated at $1 \mathrm{~mL} / \mathrm{min}$ in a mixture of solvent $\mathrm{A}$ (water/formic acid 95:5 v/v) and solvent B (acetonitrile/water/formic acid 80:15:5 v/v/v) in a 97/3 ratio. Elution was performed using a binary gradient starting from: isocratic 3\% B to $90 \%$ $\mathrm{B}$ according to a complex profile. The elution was monitored on a Waters 996 photodiode array detector. Preliminary sample preparation consisted in the solid phase extraction procedure on Sep Pak tC18 cartridges. The main dimeric and trimeric forms were identified on the basis of their retention time and on the mass signals in a LC-ESI-MS experiment.

Monomeric flavan-3-ols and oligomeric proanthocyanidins from wines, grape seeds, and skins of Graciano, Tempranillo, and Cabernet Sauvignon varieties have been identified by means of HPLC/ESI-MS data and by comparison with the retention time and spectral features of flavan-3-ol reference compounds ${ }^{29}$. The HPLC followed a preliminary fractionation by polyamide column chromatography. The equipment used for the analysis consisted of a Konik Instruments UV-vis detector (Uvis 200), a Waters 717 Plus autosampler, and a Merck Hitachi L-6200A pump, coupled to a Konikron data treatment system. Separation was performed on a reversed-phase Merck (Darmstadt, Germany) C18 Lichrosphere 100 column $(250 \mathrm{~mm}$ x $4.6 \mathrm{~mm}, 5 \mu \mathrm{m})$ at room temperature. For oligomeric procyanidins, a gradient consisting of solvent A (distilled water) and solvent B (water/acetic acid, 90/10, v/v) was applied at a flow rate of 1.0 $\mathrm{mL} / \mathrm{min}$ from $10 \%$ to $100 \% \mathrm{~B}$ (complex profile) followed by washing (methanol/water, 50/50, v/v) over a total period of $100 \mathrm{~min}$ and reequilibration of the column for $20 \mathrm{~min}$ under initial gradient conditions. Detection was performed at $280 \mathrm{~nm}$.

In the same work a Hewlett-Packard series 1100 chromatography system equipped with a diode array detector (DAD) and a quadrupole mass spectrometer (Hewlett-Packard series 1100 MSD) with an electrospray interface was used for HPLC/ESI-MS experiments. Separation was performed on a reversed-phase Waters Nova Pak C18 (300mm $\mathrm{x} 3.9 \mathrm{~mm}, 4 \mu \mathrm{m})$ column at room temperature. The solvent gradient described above for oligomeric procyanidins was applied at a flow rate of $0.7 \mathrm{~mL} / \mathrm{min}$. DAD detection was performed from 220 to $380 \mathrm{~nm}$.

Reversed phase HPLC has been used for determination of some low molecular weight proanthocyanidins in Japanese foodstuffs and grape seeds extracts ${ }^{54}$. The experiment was performed on a Shimadzu HPLC apparatus, ClassVP Series, equipped with a SPD-M10AVP diode array detector. The column was a Develosil 300 ODS-HG-5, which 
has a pore size of $300 \AA$. Unlike ordinary reversed-phase HPLC columns which has pore size of about $100 \AA$, using the column with the larger pore size made it possible to determine low molecular weight compounds (less than 1000), while higher molecular weight compounds have not been eluted. Grape seed tannins include compounds of high molecular weight (greater than 1000), therefore despite lower sensitivity the Develosil 300 column was used to determine all compounds in the studied objects.

The analysis conditions included a gradient elution performed at $35^{\circ} \mathrm{C}$. Mobile phase A was the solution of phosphoric acid in water $(1: 1000, \mathrm{v} / \mathrm{v})$ and the mobile phase B contained phosphoric acid in acetonitrile $(1: 1000, \mathrm{v} / \mathrm{v})$. Starting the gradient with pure A it was arrived at 100\% B in 61 minutes (complex profile), followed by a re-equilibration step. Detection was performed simultaneously with both DAD (set at $210 \mathrm{~nm}$ ) and fluorescence detector (ex. $283 \mathrm{~nm}$, em. $317 \mathrm{~nm}$ ). The fluorescence detection proved to be the most sensitive in this case but it does not allowed detection of gallic acid under the reported detection conditions. Phosphoric acid has an absorbance at $210 \mathrm{~nm}$, therefore the UVHPLC chromatograms were corrected by subtracting the base line. Catechin, epi-catechin, B1 (3), B2 (4) and C2 (12) compounds have been determined. The other oligomers appeared in the chromatogram as a broad unresolved band.

A very interesting example of using RP HPLC in the analysis of GST was reported recently by Santos-Buelga and collaborators. ${ }^{31}$ The methodology for the qualitative and quantitative characterization of wine proanthocyanidins was optimized using HPLC-MS. Chromatographic separations were performed both in analytical and semi-preparative versions.

The crude GST extract was fractionated on a Sephadex LH-20 column $(500 \times 30 \mathrm{~mm})$ using ethanol as a solvent. The procyanidin dimers B2 (4), B1 (3), and B2-3-O-gallate along with the trimer EEC (EC-4,8-EC-4,8-C) were isolated from the obtained fractions by semipreparative HPLC. It employed a Waters 600 series pump and a Phenomenex $5 \mu \mathrm{m}$ Ultracarb ODS20 (10x250 mm) column. The solvents were (A) acetic acid 5\% and (B) methanol. The gradient used was from pure A to B according to a stepwise profile run for $60 \mathrm{~min}$. Detection was carried out at $280 \mathrm{~nm}$, and the peaks were collected by a fraction collector. The purity and identity of the isolated GST were checked by HPLC/DAD and LC-MS by comparison with standards previously obtained in these laboratories.

For analytical purposes analyses were carried out using a Hewlett-Packard 1100 chromatograph with a quaternary pump and a diode array detector (DAD) coupled to an HP Chem Station. A Waters Spherisorb S3 ODS-2 C8, $3 \mu \mathrm{m}$ $(4.6 \times 150 \mathrm{~mm})$ column was used thermostated at $30{ }^{\circ} \mathrm{C}$. The mobile phase was (A) $2.5 \%$ acetic acid, (B) acetic acid/ acetonitrile (10:90, v:v), and (C) acetonitrile. The elution gradient was established from $100 \%$ A to $100 \% \mathrm{~B}$, followed by $15 \% \mathrm{C}$ in $\mathrm{B}$ to $50 \% \mathrm{C}$ in $\mathrm{B}$ for a total of $40 \mathrm{~min}$. Unfortunately, original chromatograms of wine samples are not provided in this paper. The results of checking the purity of individual GST are only presented.

Early separations of proanthocyanidins by normal phase chromatography were met with limited success but over time more understanding came about for example, increases in retention times were found to correspond with increasing degrees of polymerization. In the case of procyanidins, normal phase HPLC separation is based on hydrogen bonding interactions between silica hydroxyls with the larger oligomers having more extensive interactions and thus longer retention times. ${ }^{55}$

Normal phase HPLC, coupled with MALDI-TOF MS was used to characterize the proanthocyanidin composition of the grape seed extract. ${ }^{56}$ A Phenomenex Luna $5 \mu \mathrm{m}$ Silica column $(250 \times 4.6 \mathrm{~mm})$ was used for all these analyses. Detection was made by UV at $\lambda=280 \mathrm{~nm}$. The ternary mobile phase consisted of (A) dichloromethane, (B) methanol, and $(\mathrm{C})$ acetic acid and water $(1: 1 \mathrm{v} / \mathrm{v})$. A complex series of linear gradients were used in the analysis over a 70 minutes period. The flow rate was kept at $1 \mathrm{~mL} / \mathrm{min}$ and column temperature at $37^{\circ} \mathrm{C}$. The resolution of peaks was not excellent, but the most useful information provided in this paper is connected to the selective detection of certain mass fragments using the APCI in the MS detector.

A new normal phase chromatography method has been developed by Kennedy and Waterhouse. ${ }^{57}$ It uses a binary gradient with mobile phases containing methylene chloride-methanol-formic acid-water, (A) 0:97:2:1 and (B) 83:14:2:1, both containing $20 \mathrm{mM}$ heptanesulfonic acid. This method was used in a very recent paper from the same research group $^{7}$ for the analysis of GST. Additionally, a simple method to separate red wine nonpolymeric and polymeric phenols by SPE was devised. HPLC was used to monitor the obtained GST fractions. An HP 1090 apparatus with a Phenomenex Luna Silica 2 (particle size, $5 \mu \mathrm{m} ; 250 \times 4.60 \mathrm{~mm}$ i.d.) column, protected by a guard column $(10 \times 4 \mathrm{~mm})$ containing the same material was used to determine the nature of the phenolic compounds. The injection volume was $10 \mu \mathrm{L}$ for each sample. Samples were analyzed at $280 \mathrm{~nm}$. The elution conditions were as follows: $0.75 \mathrm{~mL} / \mathrm{min}$, linear gradients from 0 to $34 \% \mathrm{~A}$ in $30 \mathrm{~min}$, from 34 to $100 \% \mathrm{~A}$ in $5 \mathrm{~min}$, and 100\% A for $10 \mathrm{~min}$. The column was re-equilibrated with B for $10 \mathrm{~min}$ before subsequent injections. This method allowed separation of monomeric compounds from polymers.

Along with RP and normal phase HPLC, some papers relate on the use of gel filtration columns for analysis of GST. Gel permeation chromatography (GPC) could directly provide information on both degree of polymerization and average molecular weight. GPC analysis of proanthocyanidins has traditionally been carried out on acetylated derivatives. Derivatization was considered necessary because proanthocyanidins were too polar to be separated on currently available GPC columns. However, molecular weights determined by GPC were slightly higher compared to the ones obtained by degradation, due to the lack of suitable calibrating standards. 
Kennedy and Taylor devised a method for gel permeation chromatography of GST ${ }^{58}$ and this method was used latter on by the same authors to monitor the oxidative degradation of proanthocyanidins under basic conditions. ${ }^{2}$ This method allowed measuring the size distribution to provide additional information on how oxidation affected the size of proanthocyanidins. Unfortunately, this method allowed the separation of proanthocyanidins only as broad bands, with no separate peaks of individual compounds.

A better resolution was achieved in the work of Peterlongo and collaborators ${ }^{13}$. GPC separations were carried out at room temperature on a PL Gel column 300=7.6 mm i.d., particle size $5 \mathrm{~mm}$, pore type $500 \mathrm{~A}$ (HP) connected to a precolumn 0.5-mm filter. The elution was isocratic using THF/aqueous $\mathrm{LiBr} 1.2 \times 10^{-4} \mathrm{M}$ 95:5 at a flow rate of $1.0 \mathrm{ml} / \mathrm{min}$. Detection was at $280 \mathrm{~nm}$. Acquisition time was $15 \mathrm{~min}$. The analyzed polymers with decreasing molecular weights were eluted in succession. GPC analysis, performed as described was able not only to provide information on the composition of the extract, by evaluating the shape of the chromatographic profile, but also to allow a quantitative evaluation of the polyphenols in comparison to LeucoselectTM reference standard, thus ensuring constancy and reproducibility of biological effects.

\subsection{Mass spectrometry methods}

Mass spectrometry is a very powerful method for elucidation of the chemical structure of natural products. Its applicability is governed mostly by the ionization method employed for the transition of an uncharged inert molecule to an ion that is capable to be accelerated in the electromagnetic field. Electron impact ionization (EI) techniques historically are among the first ionization methods used in the investigation of polyphenolics from plants. It was used successfully to characterize monomeric phenolics, but its applicability to oligomers and polymers was limited because it required derivatization to increase their volatility. That's why fruit procyanidins have been acylated for EI analysis and this extra-step, along with the limited mass information available from this ionization technique severely limited its usefulness.

The development of liquid secondary ion mass spectrometry (LSI-MS) and fast atom bombardment mass spectrometry (FAB-MS) provided additional opportunities for structural identification of oligomeric polyphenolics with no need for derivatization and minimal manipulations required for the sample preparation. LSI typically uses cesium ions as the particle beam source, whereas FAB uses a neutral inert gas (argon or xenon). The sample is mixed into 1 to $2 \mu \mathrm{L}$ of glycerol or other liquid matrix and applied to the tip of a sample probe, which is then introduced into the ion source chamber. One major role of the matrix, because of its low freezing point, is to keep the sample in a liquid state as it enters the high vacuum ion source. This matrix also reduces damage to the analyte caused by the high energy bombarding particle. The subsequent bombardment causes the ejection of a desorbed secondary ion beam containing positive and negative ions in addition to neutral species. The relative abundances are controlled by the source potentials, the analyte itself, and the nature of the support matrix. This is a relatively "soft" ionization procedures that produces abundant molecular ions with minimal structural fragmentation. The major advantage of FAB is that it is easy and fast to operate, while the spectra are simple to interpret. However, one of the major disadvantages of the FAB technique is that it requires a high concentration of the organic liquid matrix (typically 80 to $95 \%$ glycerol), overall giving only moderate sensitivity. Matrix cluster ions can, in some cases, dominate the mass spectrum. In addition, damage to the matrix caused by particle bombardment gives intense chemical background. It has been shown that discrimination in ionization efficiency of one analyte over another, due to differences in hydrophobicity and surface activity, causes problems when analyzing mixtures especially in quantitative applications. That's why the spectrum must typically be obtained from relatively pure samples.

This problem was successfully overcomed with the development of matrix-assisted laser desorption ionization (MALDI) mass spectrometry. Laser desorption techniques had existed since the early 1960s; however, their mass cut-off was relatively low. Low-energy nitrogen laser light, typically at $337 \mathrm{~nm}$, in conjunction with a suitable matrix, normally a UV-absorbing organic molecule or a metal powder, could protect the analyte from degradation during the vaporization step, enabling the mass spectrometry of large biomolecules. In addition to allowing an increased mass range, MALDI has proven robust against sample contamination, although sample clean-up is still recommended. MALDI also primarily produces singly charged ions, allowing for the analysis of complex samples. When coupled to time-of-flight (TOF) spectrometers, which have no $\mathrm{m} / \mathrm{z}$ limits, MALDI is an especially powerful mapping tool. The disadvantages of this method are connected to the impossibility of quantitative analysis. Another inherent disadvantage to MALDI is the inability to conveniently couple it to chromatography: techniques to couple liquid chromatography to MALDI have proven less successful, although various approaches have been devised.

An alternative soft ionization method to MALDI, thermospray ionization was introduced in the early 80-es for the coupling of analytical HPLC at conventional flow rates to a mass spectrometer. The effluent from the HPLC column is vaporized under reduced pressure by heating a stainless steel tube of 0.10 to $0.15 \mathrm{~mm}$ inner diameter. The resulting supersonic jet contains small droplets that vaporize further due to the hot gas in this low pressure region of the ion 
source. Complete evaporation of the solvent from the liquid droplets produces gas phase ions from ionic compounds in the sample solution or from gas phase chemical ionization when an auxiliary filament or low-current discharge device is used. Ionization requires polar or charged species and volatile buffers. Thermospray is considered a soft ionization technique and induces only limited fragmentation of the analyte.

Another ionization method that has affected a tremendous impact over the last few years on the use of mass spectrometry in biological research is the electrospray ionization. It was the first method to extend the useful mass range of instruments to well over 50,000 Da. The sample is usually dissolved in a mixture of water and organic solvent, commonly methanol, isopropanol or acetonitrile. It can be directly infused, or injected into a continuous-flow of this mixture, or be contained in the effluent of an HPLC column or CE capillary. The ionization process starts with the sample nebulization step, which produces electrically charged droplets followed by liberation of the formed ions from the droplets in a combined process of solvent evaporation and Coulombic repulsion. Finally, the ions thus produced are swept from the atmospheric source region into the mass analyzer. The gradual thermal de-solvation leads to electrospray being a very "soft" ionization method. Unless the potential difference between the transfer capillary and the analyzer is increased, which results in collision-induced dissociations, there is minimal fragmentation of the analytes. An unique advantage of ESI to other methods is that the process leads to the formation of both singly and multiply charged ions and as a consequence of the multi-charging phenomenon, the instrument can be calibrated in the low $\mathrm{m} / \mathrm{z}$ range, with no need of using sophisticated TOF techniques, using singly-charged calibrants with known exact masses. Major disadvantages are that spray formation is adversely affected even by moderate buffer and salt concentrations, and that mixtures of high mass samples can give overlapping charge state distributions that may be difficult to assign to individual components.

Mass spectrometry was used as a fast and direct method for elucidating the polyphenol constituents of grape seed extracts. In particular, the composition of various commercial extracts and of oligomeric and polymeric procyanidin fractions from grape seeds were determined by liquid secondary ion mass spectrometry (LSIMS) in negative ion mode. ${ }^{59,33}$ Electrospray mass spectrometry ESI-MS was also used, directly or combined with liquid chromatography, for studying oligomeric and polymeric tannins contained in grape seeds; the negative ion ESI mass spectra showed the presence of a series of non-galloylated and galloylated oligomeric procyanidins up to trigalloylated octamer. ${ }^{33}$

During the last 7 years most of the reports on using MS methods are related to the hyphenated technique HPLCMS. ESI was the ionisation method of choice in these studies. We will provide below the most recent examples reported in the literature devoted to investigation of GST.

ESI mass spectroscopy was reported in the work of Gabetta et al. ${ }^{13}$ to identify and completely characterize the proanthocyanidin constituents of Leucoselect ${ }^{\mathrm{TM}}$ extract up to heptamers and their galates. The studied samples were injected into the spectrometer directly before and after fractionation procedure. In fact, each fraction was characterized by mass spectrum containing peaks corresponding to the protonated molecule $[\mathrm{M}+\mathrm{H}]^{+}$for each polymeric constituent. Ions at m/z 579, 867 and 1155 are due to dimers, trimers and tetramers and ions at m/z 731, 1019 and 1307 corresponding to their monogallates. Ions at $\mathrm{m} / \mathrm{z} 883,1171$ and 1459 corresponding to digallate derivatives of dimers, trimers and tetramers, respectively, and ions at m/z 1323 and 1611 corresponding to trigallate derivatives of trimers and tetramers, were also present. Fractions of higher oligomers showed ions at m/z 1443, 1731 and 2019 corresponding to pentamers, hexamers and heptamers; ions at m/z 1595, 1883 and 2171 corresponding to their monogallate derivatives; ions at $\mathrm{m} / \mathrm{z} 1747$ and 2035 corresponding to pentamer and hexamer digallate derivatives; and ions at m/z 1899 and 2187 corresponding to pentamer and hexamer trigallate derivatives. The last one was the largest polymeric proanthocyanidin detected in the mass spectra. No ions were detected above $\mathrm{m} / \mathrm{z} 2200$, or double charge peaks over the whole mass range. Ions corresponding to the loss of flavan-3-ol units were also present, showing that a certain degree of fragmentation took place even in ESI-MS conditions.

The thermospray ionization was also employed in this work in tandem with HPLC separation. Positive mass spectra have been acquired from m/z 160 to 1200 over a scan time of $1 \mathrm{~s}$. Unlike ESI, TSI allowed only detection of monomeric flavan-3-ols and dimeric proanthocyanidins. The spectra contain only few ions, but they are useful for the identification. In this case catechin and epicatechin gave only the protonated molecule $[\mathrm{M}+\mathrm{H}]^{+}$at $\mathrm{m} / \mathrm{z}=291$. Dimers give the protonated molecule $[\mathrm{M}+\mathrm{H}]^{+}$at $\mathrm{m} / \mathrm{z}=579$, base peak being the flavan-3-ol protonated unit $\mathrm{m} / \mathrm{z}=291$. The presence of a peak at $\mathrm{m} / \mathrm{z}=601$ corresponding to the $[\mathrm{M}+\mathrm{Na}]^{+}$ion, supports the molecular weight attribution. The dimer gallate gives a positive TSP mass spectrum showing, besides the protonated molecule $[\mathrm{M}+\mathrm{H}]^{+}$at $\mathrm{m} / \mathrm{z}=731$, the ions corresponding to the loss of gallic acid residue at $\mathrm{m} / \mathrm{z}=579$ and to the loss of a flavan-3-ol unit at $\mathrm{m} / \mathrm{z}=443$.

Matrix-assisted laser desorption/ionization time-of-flight mass spectrometry was used to characterize the procyanidin composition of a grape seed extract. ${ }^{56}$ Oligomers up to nonamers were observed. HPLC/ESI-MS was shown to separate the procyanidin isomers of the lower degree of polymerization but failed to resolve oligomers larger than pentamers. MALDI-TOF MS, on the other hand, allowed a rapid analysis of such complex mixtures. The individual oligomers of procyanidins in grape seeds were well resolved in MALDI-TOF MS spectra with their molecular weight determined with great accuracy. The dominating features of the spectra are two major series of ions separated by $152 \mathrm{Da}$. The first series was attributed to the sodium adducts of monomers and the oligomers composed purely of $(+)$-catechin and (-)-epicatechin, with the molecular weight of $290+288(\mathrm{n}-1)$, n being the degree of polymerization. The second 
series consists of ions with the molecular weight of $442+288$ (n - 1), which are the sodium adducts of oligomers containing one galloylated unit, i.e., either a $(+)$-catechin gallate or an (-)-epicatechin gallate. Sodium was shown to originate from the grape seeds themselves and its presence greatly enhances the signal sensitivity.

The potential of the MALDI-TOF MS technique as a quantification tool was also discussed. Conditions for MALDI-TOF in terms of matrix selection and sample preparation have been optimized. 2,5-dihydroxybenzoic acid was shown to be the optimal matrix providing the broadest mass range with the least background noise. The relative intensity (sample intensity/internal standard intensity) of each oligomer showed a general increasing trend with increasing concentration. Application of MALDI-TOF as a quantitation tool is hampered though by the absence of individual standards of higher grape seeds proanthocyanidins.

Fractionated GST and individual compounds separated by RP HPLC were examined by electrospray Ion-Trap Mass Spectrometry in the negative ion mode. ${ }^{8}$ The electrospray matrix was $80 \% \mathrm{MeOH} / 20 \% \mathrm{H}_{2} \mathrm{O}$. In some cases MS/MS was also run on individual peaks in order to determine the proanthocyanidin structure of the compounds. Primary spectra showed categorical predomination of the molecular peaks $[\mathrm{M}-\mathrm{H}]^{-}$, and it was proved on an example of an individual HPLC peak, whyle MS/MS experiment provided a deeper fragmentation of the analized oligomer. Compounds up to pentamers ( $\mathrm{m} / \mathrm{z} 1441)$ have been detected in the analized extract.

A recent paper provided data concerning HPLC/ESI-MS fragmentation patterns of oligomeric procyanidins in grape and wine fractions obtained by polyamide column chromatography. ${ }^{29}$ Along with the most abundant peaks of molecular ions $[\mathrm{M}-\mathrm{H}]^{-}($dimer $\mathrm{m} / \mathrm{z} 577$, dimer gallate $\mathrm{m} / \mathrm{z} 729$, trimer $\mathrm{m} / \mathrm{z} 865)$, some peaks attributed to Retro-DielsAlder (RDA) fission fragments originated from dimeric and trimeric procyanidins (ions with $\mathrm{m} / \mathrm{z} 425 \mathrm{and} \mathrm{m} / \mathrm{z} 713$ ). An ion corresponding to its subsequent water elimination was only detected for dimeric procyanidins (m/z 407). Under the reported MS conditions, trimeric procyanidins only underwent one-stage RDA fission, since the characteristic ions (m/z 425 and 407) that resulted for the dimeric procyanidins were not observed. Some other MS fragments were tentatively attributed to different paths of fragmentation of different oligomers (up to trimers).

Although the molecular peaks in the ESI spectra are the most abundant, examining of minor ones also leads to very important conclusions about the GST structure. They can show additional information about the fragmentation path and sometimes the minor peaks correspond to multiple charged ions. This observation has led to important conclusions made in a recent work connected to the investigation of GST degree of polimerization. ${ }^{24}$ Two fractions originated from a grape seeds extract were directly injected into the mass spectrometer under ESI conditions. The mean degree of polymerization of the investigated fractions, calculated on the basis of thyolisis experiments was 3 and 9 respectively. As it was expected, in the spectra acquired on the entire mass range (200-3000 Da) predominated the molecular ions of anthocyanidins and their galates, up to heptamers. The mean degree of polymerization (mDP) calculated on the basis of the MS experiment was in accordance with the thyolisis data for the fraction with the $\mathrm{mDP}=3$. For the fraction with the $\mathrm{mDP}=9$ there was a discrepancy between MS and thyolisis data. According to mass spectrum of this fraction, for the single charged series of molecular peaks $[\mathrm{M}-\mathrm{H}]^{-}$the most abundant ions corresponded to proanthocyanidins with degree of polymerization 3 or 4 , much lower than that estimated from acid hydrolysis $(\mathrm{mDP}=8.9)$. A careful examination of the minor peaks in the spectrum of the fraction with $\mathrm{mDP}=9$ showed that numerous ions could be assigned as doubly charged, based on the mass difference between isotopic peaks and these peaks were absent in the spectrum of the first fraction $(\mathrm{mDP}=3)$. Taking into consideration these ions, it was found that the highest value of polymerisation degree for this fraction was 13 . Recording the spectra in a narrower $\mathrm{m} / \mathrm{z}$ range with a higher resolution led to detection of triple charged ions $[\mathrm{M}-3 \mathrm{H}]^{3-}$ having the maximum $\mathrm{m} / \mathrm{z}=2890.5$ that corresponds to the DP 28 with 4 galloylation and $\mathrm{m} / \mathrm{z}$ $=2947.7$ that corresponds to the DP 27 with 7 galloylation units. The triple charged ions appeared to be eclipsed by more abundant single charged ones under low resolution condition of spectra aquisition.

The conclusion made in above discussed paper $^{24}$ has been confirmed by other studies of the low-molecular weight proanthocyanidine fraction isolated from wine samples. In their recent work Santos-Buelga and colaborators ${ }^{3}$ used ESI MS to monitor the proanthocyanidin composition of wines at different maceration stages. Unlike in other studies, ionization was performed in the positive mode. No multiple charged ions have been detected and the molecular ions prevailed categorically over other fragments. Proanthocyanidins up to hexamers have been detected in the wine samples, even at advanced maceration stages.

In conclusion, we shall mention that mass spectrometry is a powerful method for investigation of grape seeds proanthocyanidins. Application of soft ionization techniques like MALDI-TOF or ESI allows monitoring exclusively molecular ions both in negative and positive modes. Higher molecular oligomers can be ionized more easily to produce multiple charged ions which can be distinguished by the distance between the isotopic peaks of the carbon. ${ }^{60}$

\subsection{Miscellaneous methods}

Investigation of grape seed proanthocyanidins included also other physicochemical methods, like thin layer chromatography (TLC) and Nuclear Magnetic Resonance (NMR) spectroscopy. But their power and usefulness are less satisfactory, compared to HPLC and MS methods. 
The value of TLC is given by its simplicity, low cost and rapidity of analysis. Most of the recently reported examples of using TLC in the investigation of GST relate only to normal phase variant. ${ }^{15,538,61}$ The resolution power of TLC, even on HPTLC plates, allows separation of proanthocyanidin mixture groups by molecular weight as bands. Consequently, the method can be successfully used to monitor preliminary fractionation of the natural extracts. In our opinion this method has a further potential, due to the continuous evolution of the stationary phases, especially in the reversed phase variant.

Nuclear Magnetic Resonance spectroscopy (NMR) has been used as a powerful tool in the structure elucidation of proanthocyanidins. But aplication of NMR, even at high operating frequencies required preliminary separation of individual compounds that is not always a trivial task. One of the first work in this field relates using of ${ }^{1} \mathrm{H}$ NMR to estimate the polymerization degree of proanthocyanidin mixtures. ${ }^{62}{ }^{1} \mathrm{H}$ and ${ }^{13} \mathrm{C}$ NMR spectroscopy techniques were used to estimate the degree of polymerization. In the absence of doubly linked A-type bonds, the average molecular weight can be determined directly from the ${ }^{13} \mathrm{C}$ NMR spectra by comparing the areas of the $\mathrm{C} 3$ resonances of the terminal and extender flavan-3-ol units, at 67-68 and 72-73 ppm, respectively.

A following report shows a complete and unambiguous assignment of the ${ }^{1} \mathrm{H}$ and ${ }^{13} \mathrm{C} \mathrm{NMR}$ spectra of peracetylated catechin-(4-8)-catechin-(4-8)-catechin procyandin trimer, accomplished by reverse two-dimensional chemical shift correlation methods. ${ }^{63}$ Two-dimensional NMR was the only analytical tool which used to check the identity of grapevine species, cultivars or clones. ${ }^{64}$ The structure of the proanthocyanidin B-2 dimer was proved by two-dimensional heteronuclear NMR spectroscopy. ${ }^{65}$ Identification of the peracetylated dimer B2 was also performed by ${ }^{1} \mathrm{H}$ and ${ }^{13} \mathrm{C}$ NMR. ${ }^{5}$

Another example of using of ${ }^{1} \mathrm{H}$ NMR to estimate the polymerization degree of proanthocyanidin mixtures was reported by Guyot and colaborators. ${ }^{66}$ It was shown that by integrating the A-ring proton signals between 5.8 and 6.5 ppm in ${ }^{1} \mathrm{H}$ NMR spectra and comparing them to the intensity of the H4 signals of the terminal units between 2.4 and 3.0 ppm it is possible to derive the DP. This strategy was used latter by Schmidt and collaborators. ${ }^{61}$

Various phenolic compounds were synthesized in an aqueous-alcoholic solution containing $(+)$-catechin and glyoxylic acid which was used as a model of fruit-derived food browning that usually occurs during aging. ${ }^{67}$ After purification by semi-preparative HPLC, the isolated compounds were subjected to homo- and heteronuclear proton and carbon NMR analysis including COSY, TOCSY, ROESY, HSQC and HMBC techniques. These experiments allowed the structural elucidation and complete ${ }^{1} \mathrm{H}$ and ${ }^{13} \mathrm{C}$ NMR assignment of the isolated compounds. The structure and conformation of two native procyanidin trimers in water have been determined using 2D NMR and molecular mechanics. ${ }^{68}$

\section{CONCLUSIONS}

To date, investigation of the structures of grape proanthocyanidins has been performed in different ways depending on the extension of the polymeric chain. In fact, only low molecular weight tannins up to tetramers can be isolated and characterized as pure compounds. Isolation of pure polymeric tannins is difficult. In most cases, only fractions corresponding to mixtures of several polymers can be obtained. Chemical methods must therefore be developed to separate individual proanthocyanidin molecules for identification while retaining them unchanged for simultaneous bioactivity testing. This problem represents a real challenge to chemical community.

\section{Acknowledgements}

This work was performed within the INTAS project 05-104-7505.

\section{REFERENCES}

[1] Vidal, S.; Cartalade, D.; Souquet, J.M.; Fulgrand, H.; Cheinier, V. Changes in Proanthocyanidin Chain Length in Winelike Model Solutions. J. Agric. Food Chem. 2002, 50, 2261-2266.

[2] Jorgensen, E.M.; Marin, A.B.; Kennedy, J. A. Analysis of the Oxidative Degradation of Proanthocyanidins under Basic Conditions. J. Agric. Food Chem. 2004, 52, 2292-2296.

[3] Gonzalez-Manzano, S.; Santos-Buelga, C.; Perez-Alonso, J.J.; Rivas-Gonzalo, J.C.; Escribano-Bailon, M.T. Characterization of the Mean Degree of Polymerization of Proanthocyanidins in Red Wines Using Liquid Chromatography-Mass Spectrometry (LC-MS). J. Agric. Food Chem. 2006, 54, 4326-4332.

[4] Peyrot des Gachons, C.; Kennedy, J. A. Direct method for determining seed and skeen proanthocyanidins extraction into red wine. J. Agric. Food Chem. 2003, 51, 5877-5881.

[5] de Freitas, V.; Glories, Y.; Laquerre, M. Incidence of Mollecular Structure in Oxidation of Grape Seeds Procyanidins. J. Agric. Food Chem. 1998, 46, 376-382. 
[6] Uchida, S.; Edamatsu, R.; Hiramatsu, R.; Mori, A.; Nonaka, G.I.; Nishioka, I.; Ozaki, M. Condensed tannins scavenge active oxygen free radicals. Med. Sci. Res. 1987, 15, 831-832.

[7] Pinelo, M.; Laurie, V.F.; Waterhouse, A.L. A Simple Method To Separate Red Wine Nonpolymeric and Polymeric Phenols by Solid-Phase Extraction. J. Agric. Food Chem. 2006, 54, 2839-2844.

[8] Fitzpatrick, D.F.; Fleming, R.C.; Bing, B.; Maggi, D.A.; O’Malley, R.M. Isolation and Characterization of Endothelium-Dependent Vasorelaxing Compounds from Grape Seeds. J. Agric. Food Chem. 2000, 48, 63846390.

[9] Santos-Buelga, C.; Scalbert, A. Proanthocyanidins and Tannin-like Compounds - Nature, Occurrence, Dietary Intake and Effect on Nutrition and Health. J. Sci. Food Agric. 2000, 80, 1094-1117.

[10] Murray M, Pizzorno J. Procyanidolic oligomers. In: Murray M, Pizzorno J, eds. The Textbook of Natural Medicine. 2nd ed. London: Churchill Livingston; 1999, 899-902.

[11] Pekic, B.; Kovac, V.; Alonso, E.; Revilla, E. Study of the Extraction of proanthocyanidins from grape seeds. Food Chem. 1998, 61(1-2), 201-206.

[12] Teissedre, P.L.; Waterhouse, A.L.; Frankel, E.N. Principal Phenolic Phytochemical in French Syrah and Grenache Rhone Wines and Their Antioxidant Activity in Inhibiting Oxydation of Human Low Density Lypoproteins. $J$. Inter. Sci. de la Vigne et du Vin. 1995, 29(4), 205-212.

[13] Gabetta, B.; Fuzzati, N.; Griffini, A.; Lolla, E.; Pace, R.; Ruffilli, T.; Peterlongo, F. Characterization of proanthocyanidins from grape seeds. Fitoterapia. 2000, 71, 162-175.

[14] da Silva J, Rigaud J, Cheynier V, et al. Procyanidin dimers and trimers from grape seeds. Phytochemistry. 1991, 30, 1259-1264.

[15] Oszmianski, J.; Sapis, J.C. Fractionation and Identification of Some Low Molecular Weight Grape Seed Phenolics. J. Agric. Food Chem. 1989, 37, 1293-1297.

[16] Lea, A.G.H.; Bridle, P.; Timberlake, C.F.; Singleton, V. The procyanidin of white grapes and wines. Am. J. Enol. Vitic. 1979, 30, 289-300.

[17] Romeyer, F.M.; Macheix, J.; Sapis, J. Changes and importance of oligomeric procyanidins during maturation of grape seeds. Phytochemistry. 1986, 25, 219-221.

[18] da Silva, R.J.M.; Bourzeix, M.; Cheynier, V.; Moutounet, M. Procyanidin composition of Chardonnay, Mauzac and Grenache blanc grapes. Vitis. 1991, 30, 245-252.

[19] da Silva, R.J.M.; Rosec, J.P.; Bourzeix, M.; Mourgues, J.; Moutounet, M. Dimer and trimer procyanidins in Carigan and Mourvedre grapes and red wines. Vitis. 1992, 31, 55-63.

[20] Santos-Buelga, C.; Francia-Aricha, E.M.; Escribano-Bailon, M.T. Comparative flavan-3-ol composition of seeds from different grape varieties. Food Chem. 1995, 53, 197-201.

[21] Kovac, V.; Bourzeix, M.; Heredia, N.; Ramos, T. Etude des catechines et proanthocyanidols de raisin et vins blancs. Revue France d'Oenologie. 1990, 125, 7-14.

[22] Kovac, V.; Alonso, E.; Revilla, E. The effect of adding supplementary quantities of seeds during fermentation on the phenolic composition of wines. Am. J. Enol. Vitic. 1995, 46, 363-367.

[23] de Freitas, V.; Glories, Y.; Bourgeois, G.; Vitry, C. Characterisation of oligomeric and polymeric procyanidins from grape seeds by liquid secondary ion mass spectrometry. Phytochemistry, 1998, 49, 1435.

[24] Hayasaka, Y.; Waters, E.J. Cheynier, V.; Herderich, M.J.; Vidal, S. Characterization of Proanthocyanidins in Grape Seeds Using Electrospray Mass Spectrometry. Rapid Commun. Mass Spectrom. 2003, 17, 9-16.

[25] Sun, B.S.; Leandro, C.; da Silva J.M.R.; Spranger, I. Separation of Grape and Wine Proanthocyanidins According to their Degree of Polymerization. J. Agric. Food Chem. 1998, 46, 1390-1396.

[26] Labarbe, V.; Cheinier, V.; Brossaud, F.; Souquet, J.M.; Moutounel, M. Quantitative fractionation of grape proanthocyanidins according to their degree of polymerization. J. Agric. Food Chem. 1999, 47, 2719-2723.

[27] Michaud, J.; Lacaze, P.; Masquelier, J. Fractionement des oligomers flavanolique du raisin. Bull. Soc. Pharm. Bordeau. 1971, 110, 111-116.

[28] Nonaka, G.J.; Miwa, N.; Nishioka, I.; Stilbene glycoside gallates and proanthocyanidins from Polygonum multiflorum. Phytochemistry. 1992, 21(2), 429-432.

[29] Monagas, M.; Gomez-Cordovez, C.; Bartolomea, B.; Laureano, O.; Ricardo da Silva, J.M. Monomeric, Oligomeric, and Polymeric Flavan-3-ol Composition of Wines and Grapes from Vitis vinifera L. Cv. Graciano, Tempranillo, and Cabernet Sauvignon. J. Agric. Food Chem. 2003, 51, 6475-6481.

[30] Geny, L.; Saucier, C.; Bracco, S.; Daviaud, F.; Glories, I. Composition and cellular localization of tannins in grape seeds during maturation. J. Agric. Food Chem. 2003, 51, 8051-8054.

[31] Darne, G.; Madero, T.J. Mise au point d'une methode d'extraction des lipides solubles totaux, des glusides solubles totaux et des composes phenoliques solubles totaux des organes de la vigne. Vitis. 1979, 18(3), 221228.

[32] Kuliev, R.Z.; Akhmedov, U.; Khalmatov, Kh.Kh.; Kuliev, Z.A. Dimeric Proanthocyanidins from Rhodiola semenovii. Chem. Nat. Comp. 2004, 40 (1), 94-95. 
[33] Kantz, K.; Singleton, V.L. Isolation and Determination of Polymeric Polyphenols using Sephadex LH-20 and Analysis of Grape Tissue Extracts. Am. J. Enol. Vitic. 1990, 41, 223-228.

[34] Kantz, K.; Singleton, V.L. Isolation and Determination of Polymeric Polyphenols in Wines using Sephadex LH20. Am. J. Enol. Vitic. 1991, 42, 309-316.

[35] da Silva J.M.R.; Rosec, J.P.; Bourzeix, M.; Heredia, N. Separation and Quantitative Determination of Grape and Wine Procyanidines by High Performance Reversed Phase Liquid Chromatography, J. Sci. Food Agric. 1990, 53, 85-92.

[36] Souquet, J. M.; Cheynier, V.; Brossaud, F.; Moutounet, M. Polymeric Proantocyanidines from Grape Skins. Phytochemistry. 1996, 43, 509-512.

[37] Prieur, C.; Rigaud, J.; Cheynier, V.; Moutounet, M. Oligomeric and polymeric procyanidins from grape seeds. Phytochemistry. 1994, 36, 781-784.

[38] Saucier, C.; Mirabel, M.; Daviaud, F.; Longieras, A.; Glories, Y. Rapid fractionations of grape seeds proanthocyanidins. J. Agric. Food Chem. 2001, 49, 5732-5735.

[39] Kennedy, J.A.; Matthews, M.A.; Waterhouse, A.I. Changes in grape seed polyphenol during fruit ripening. Phytochemistry. 2000, 55, 77-85.

[40] Kennedy, J.A.; Jones, G.P. Analysis of proanthocyanidins cleavage products following acid catalysis in the presence of excess floroglucinol. J. Agric. Food Chem. 2001, 49, 1740-1746.

[41] Porter, L.J.; Hrstich, L.N.; Chan, B.G. The conversion of procyanidins and prodelphinidins to cyanidin and delphinidin. Phytochemistry. 1986, 25, 223-230.

[42] Wulf, L.W.; Nagel, C.W. Analysis of phenolic acids and flavonoids by high-pressure liquid chromatography. $J$. Chromatogr. 1976, 116, 271-279.

[43] Wulf, L.W.; Nagel, C.W. High-pressure liquid chromatographic separation of anthocyanins of Vitis vinifera. Am. J. Enol. Vitic. 1978, 29, 42-49.

[44] Nagel, C.W.; Wulf, L.W. Changes in anthocyanins, flavonoids and hydroxycinnamic acid esters during fermentation and aging of Merlot and Cabernet Sauvignon. Am. J. Enol. Vitic. 1979, 30, 111-116.

[45] Lea, A.G.H. High performance liquid chromatography of cider procyanidins. J. Sci. Food Agric. 1979, 30, 833838.

[46] Lea, A.G.H. Reversed-phase gradient high-performance liquid chromatography of procyanidins and their oxidation products in ciders and wines, optimized by Snyder's procedures. J. Chromatogr. 1980, 194, 62-68.

[47] McCloskey, L.P.; Yengoyan, L.S. Analysis of anthocyanins in Vitis vinifera wines and red colour versus aging by HPLC and spectrophotometry. Am. J. Enol. Vitic. 1981, 32, 257-261.

[48] Merken HM, Beecher GR. Measurement of food flavonoids by high-performance liquid chromatography: A review. J. Agric. Food Chem. 2000, 48, 577-599.

[49] Lazarus SA, Adamson GE, Hammerstone JF, Schmitz HH. High-performance liquid chromatography/mass spectrometry analysis of proanthocyanidins in foods and beverages. J. Agric. Food Chem. 1999, 47, 36933701.

[50] de Pascual-Teresa S, Santos-Buelga C, Rivas-Gonzalo JC. Quantitative analysis of flavan-3-ols in Spanish foodstuffs and beverages. J. Agric. Food Chem. 2000, 48, 5331-5337.

[51] Carando S, Teissedre PL, Rascual-Martinez L, Cabanis JC. Levels of flavan-3-ols in French wine. J. Agric. Food Chem. 1999, 47, 4161-4166.

[52] Fuleki T, da Silva R.J.M. Catechin and procyanidin composition of seeds from grape cultivars grown in Ontario. J. Agric. Food Chem. 1997, 45, 1156-1160.

[53] Peng Z, Hayasaka Y, Iland PG, Sefton M, Hoj P, Waters EJ. Quantitative analysis of polymeric procyanidins (tannins) from grape (Vitis vinifera) seeds by reverse phase high-performance liquid chromatography. J. Agric. Food Chem. 2001, 49, 26-31.

[54] Nakamura, Y.; Tsuji, S.; Toogai, Y. Analysis of Proanthocyanidins in Grape Seed Extracts, Health Foods and Grape Seed Oils. Journal of health Science. 2003, 49 (1), 45-54.

[55] Waterhouse AL, Ignelzi S, Shirley JR. A comparison of methods for quantifying oligomeric proanthocyanidins from grape seed extracts. Am. J. Enol. Vitic. 2000, 51, 383-389.

[56] Yang, Y; Chien, M. Characterization of Grape Procyanidins Using High-Performance Liquid Chromatography/ Mass Spectrometry and Matrix-Assisted Laser Desorption/Ionization Time-of-Flight Mass Spectrometry. $J$. Agric. Food Chem. 2000, 48, 3990-3996.

[57] Kennedy, J.A.; Waterhouse, A.L. Analysis of Pigmented High Molecular-Mass Grape Phenolics Using Ion-pair, Normal-Phase High-Performance Liquid Chromatography. J. Chromatogr. A. 2000, 866, 25-34.

[58] Kennedy, J. A.; Taylor, A. W. Analysis of proanthocyanidins by high-performance gel permeation chromatography. J. Chromatogr. A. 2003, 995, 99-107.

[59] Vivas, N.; Bourgeois, G.; Vitry, C.; Glories, Y.; de Freitas, V. Determination of the composition of commercial tannin extracts by liquid secondary ion mass spectrometry (LSIMS). J. Sci. Food Agric. 1996, 72, 309. 
[60] Cheynier, V.; Doco, T.; Fulcrand, H.; Guyot, S.; Le Roux, E.; Souquet, J. M.; Rigaud, J.; Moutounet, M. ESI-MS analysis of polyphenolic oligomers and polymers. Analysis. 1997, 25, 32-37.

[61] Schmidt, B.M.; Howell, A.B.; McEniry, B.; Knight, C.T.; Seigler, D.; Erdman, J.W. JR.; Lila, M.A. Antiadhesion Components from Wild Blueberry (Vaccinium angustifolium Ait.) Fruits. J. Agric. Food Chem. 2004, 52, 64336442.

[62] Czochanska, Z.; Foo, L. Y.; Newman, R. H.; Porter, L. J. Polymeric proanthocyanidins. Stereochemistry, structural units and molecular weight. J. Chem. Soc., Perkin Trans. 1. 1980, 1, 2278-2286.

[63] Balas, L.; Vercauteren, J.; Laguerre, M. 2D NMR structure elucidation of proanthocyanidins: The special case of the catechin-(4-8)-catechin-(4-8)-catechin trimer. Magn. Reson. Chem. 1995, 33, 85-94.

[64] Forveille, L.; Vercauteren, J.; Rutledge, D.N. Multivariate statistical analysis of two-dimensional NMR data to differentiate grapevine cultivars and clones. Food Chemistry, 1996, 57, 3, 441-450.

[65] Khan, M.L.; Haslam, E.; Williamson, M.P. Structure and conformation of the procyanidin B-2 dimer. Magn. Reson. Chem. 1997, 35, 854-858.

[66] Guyot, S.; Le Guerneve, C.; Marnet, N.; Drilleau, J. F. Methods for determining the degree of polymerization of condensed tannins: A new ${ }^{1} \mathrm{H}$ NMR procedure applied to cider apple procyanidins. In Plant Polyphenols 2: Chemistry, Biology, Pharmacology, Ecology; Gross, Ed.; Kluwer Academic/Plenum Publishers: New York, 1999.

[67] Es-Safi, N.E.; Le Guerneve, C.; Cheynier, V.; Moutounet, M. 2D NMR analysis for unambiguous structural elucidation of phenolic compounds formed through reaction between (+)-catechin and glyoxylic acid. Magn. Reson. Chem. 2002, 40, 693-704.

[68] Fouquet, E.; Laguerre, M.; Pianet, I. Structural and conformational analysis of two native procyanidin trimers. Magn. Reson. Chem. 2007, 45, 157-166. 